\title{
Editions Consulted
}

This list includes primary sources quoted or cited in the text. In quoting from Latin, Old Norse-Icelandic, and Old French works, I have often ignored paragraph and chapter divisions. Unless otherwise indicated, all translations appearing in the text but not on this list are my own.

IF = Íslenzk fornrit editions, vols. 1-14 and 26-28 (Reykjavík: Hio íslenzka fornritafélag, 1933-68).

Adam of Bremen. Bernhard Schmeidler, ed., Adam von Bremen, Hamburgische Kirchengeschichte [Gesta hammaburgensis ecclesiae pontificum], Scriptores rerum germanicarum, 3d ed. (Hanover: Hahn, 1917).

Ad Herennium. Harry Caplan, ed., Rhetorica ad Herennium, Loeb Classical Library (Cambridge, Mass.: Harvard University Press, 1954).

ÁGrIP. Fínnur Jónsson, ed., Ágrip af Nóregs konunga sogum, Altnordische Saga-bibliothek, 18 (Halle/Saale: Niemeyer, 1929).

Alcuin. Karl Halm, ed., Disputatio de rhetorica et de virtutibus sapientissimi regis Karli et Albini magistri, Rhetores latini minores (Leipzig, 1863).

Wilhelm Wattenbach and Ernst Ludwig Dümmler, eds., Alcuini vita sancti Willibrordi, Bibliotheca rerum germanicarum, 6 (Berlin: Weidmann, 1878).

Aliscans. Erich Wienbeck, Wilhelm Hartnacke, and Paul Rasch, eds., Aliscans: Kritischer Text (Halle/Saale: Niemeyer, 1903).

Amadis de Gaula. Edwin B. Place, ed., Amadís de Gaula, 4 vols. (Madrid: Consejo Superior de Investigaciones Científicas, 1959-69). 
Edwin B. Place and Herbert C. Behm, trs., Amadis of Gaul (Lexington, Kentucky: University of Kentucky Press, 1974).

ARIosto. Santorre Debenedetti, ed., Lodovico Ariosto: Orlando furioso (Bari: Laterza, 1928).

ARistotle. W. D. Ross, ed., The Works of Aristotle Translated into English, 12 vols. (Oxford: Clarendon, 1906-52).

ARI PoRgiLsSon. IF I (1968).

ÁrNa saga BiskuPs. Jón Sigurð̌sson and Guð̋randr Vigfússon, eds., Biskupa sögur, 2 vols. (Copenhagen: Møller, 1858-1878), vol. I.

BandamanNa Saga. IF VII (1936).

Beowulf. Fr. Klaeber, ed., Beowulf and the Fight at Finnsburg, 3d ed. (Boston: D. C. Heath, 1950).

BÉRoul. Ernest Muret, ed., Le roman de Tristan, poème du XII siècle par Béroul, 4th rev. ed., Les classiques français du moyen âge, 12 (Paris: Champion, 1967).

Bósa saga. Otto Luitpold Jiriczek, ed., Die Bósa-saga in zwei Fassungen, nebst Proben aus den Bósa-rímur (Strasbourg: Trübner, 1893).

Brennu-Nuáls saga. See Njals saga.

Chanson de Guillaume. Duncan McMillan, ed., La chanson de Guillaume, Société des anciens textes français (Paris: Picard, 1949-50).

Chanson de Roland. Gerard J. Brault, ed. and tr., The Song of Roland: An Analytical Edition, 2 vols. (University Park, Pa.: Pennsylvania State University Press, 1978).

Chaucer, Geoffrey, F. N. Robinson, ed., The Works of Geoffrey Chaucer, 2d ed. (Boston: Houghton Mifflin, 1957).

La Chevalerie d'Ogier de Danemarche. Mario Eusebi, ed., La chevalerie d'Ogier de Danemarche (Milan: Cisalpino, 1963).

Chrétien de Troyes. Alexandre Micha, ed., Cligès (Paris: Champion, 1957).

Mario Louis Roques, ed., Erec et Enide, Les classiques français du moyen age, 80 (Paris: Champion, 1953).

Alfons Hilka, ed., Der Percevalroman (Li contes del graal) (Halle/Saale: Niemeyer, 1932).

. Mario Louis Roques, ed., Le chevalier au lion (Yvain), Les classiques français du moyen age, 89 (Paris: Champion 1960).

W. W. Comfort, tr., Chrétien de Troyes: Arthurian Romances (New York: Dutton, 1975).

Codex Regius. See Edda.

Droplaugarsona saga. IF XI (1950).

EdDa. Gustav Neckel and Hans Kuhn, eds., Edda: Die Lieder des Codex Regius nebst verwandten Denkmälern (Heidelberg: Carl Winter, 1962). 
EgILs SAGa. IF II (1933).

EILhaRT. Hadumod Bussmann, ed., Tristant von Eilhart von Oberg. Synoptischer Druck der ergänzten Fragmente mit der gesamten Parallelüberlieferung, Altdeutsche Textbibliothek, 70 (Tübingen: Niemeyer, 1969).

EIRSPENNILl. See Sverris saga.

EREX saga. Gustaf Cederschiöld, ed., Erex saga, Samfund til udgivelse af gammel nordisk litteratur, III (Copenhagen: Møller, 1880).

Foster W. Blaisdell, ed., Erex saga artuskappa, Editiones Amamagnæanæ, Series B, vol. 19 (Copenhagen: Munksgaard, 1965).

Estoire Del SAINT GRaAL. See Vulgate Cycle.

EYRBYGGJA SAGA. IF IV (1935).

Fagrskinna. Finnur Jónsson, ed., Fagrskinna. Nóregs kononga tal (Copenhagen: Møller, 1902-3).

Flateyjarbók. Guơbrandur Vigfússon and C. R. Unger, Flateyjarbók, 3 vols., Norsk historisk kildeskriftfonds skriften, 4 (Oslo: P. T. Malling, 1860-68).

Sigurour Nordal, ed., Flateyjarbók, 4 vols. (Akranes: Prentverk Akranes, 1944-45).

Floris AND Blancheflour. Walter French and Charles B. Hale, eds., Middle English Metrical Romances (New York: Prentice-Hall, 1930).

FósTBREĐra SAGA. IF VI (1943).

Froissart. Simeon Luce et al., eds., Froissart: Chroniques, 15 vols., Société de l'histoire de France (Paris: Renouard, 1869-1975).

Gamelyn. Walter French and Charles B. Hale, eds., Middle English Metrical Romances (New York: Prentice-Hall, 1930).

Gautreks saga. Wilhelm Ranisch, ed., Gautreks saga, Palaestra, 11 (1900).

Gawain and the Green Knight. J. R. R. Tolkien and E. V. Gordon, eds., Sir Gawain and the Green Knight, 2d rev. ed. (Oxford: Clarendon, 1968).

Geoffrey of Vinsauf. Documentum de modo et arte dictandi et versificandi and Poetria nova, in Edmond Faral, eds., Les arts poétiques $d u X^{\prime} I^{e}$ et du XIII ${ }^{e}$ siècle (Paris: Champion, 1958).

. Jane Baltzell Kopp, tr., Poetria nova, in James J. Murphy, ed., Three Medieval Rhetorical Arts (Berkeley: University of California Press, 1971).

GísLa SAGA. IF VI (1943).

Great Saga of Saint OLÁfr. Oscar Albert Johnsen and Jón Helgason, eds., Den store saga om Olav den hellige efter pergamenthåndskrift $i$ Kungliga Biblioteket $i$ Stockholm Nr. $24^{\text {to }}$ med varianter fra andre håndskrifter, 2 vols. (Oslo: Jacob Dybwad, 1930-33).

Grettis SAGA. IF VII (1936). 
Guillaume D'Angleterre. Maurice Wilmotte, ed., Guillaume d'Angleterre: Roman du XII ${ }^{e}$ siècle, Les classiques français du moyen age (Paris: Champion, 1927).

GunNlaugs Saga. ÍF III (1938).

HaLlFreĐAR SAGa. If $F$ VIII (1939).

Hávariar saga Ísfirdings. ÍF VI (1943).

Heidarviga Saga. ÍF III (1938).

HeIMSKRINGLA. IF XXVI-XXVIII (1941-51).

Historre de SAint Louis. See Joinville.

Historia Norwegie. Gustav Storm, ed., Monumenta historica Norvegiæ (Christiania: Brøgger, 1880).

Hensa-PóRIS SAga. Í $F$ III (1938).

Horace. H. Rushton Fairclough, Horace: Satires, Epistles, and Ars poetica, Loeb Classical Library (Cambridge, Mass.: Harvard University Press; London: Heinemann, 1929, rpt. 1970).

HrafNKels SAGa. I $F$ XI (1950).

Hugh of ST. Victor. Charles Henry Buttimer, ed., Didascalion de studio legendi, Studies in Medieval and Renaissance Latin, 10 (Washington, D.C.: Catholic University Press, 1939).

IsLENDingabók. See Ari Porgilsson.

IsLENDingasaga. See Sturlunga saga.

John of Garland. Poetria, in Edmond Faral, ed., Les arts poétiques du $X I{ }^{e}$ et du XIII ${ }^{e}$ siècle (Paris: Champion, 1958).

JoInville. Natalis de Wailly, ed., Jean de Joinville: Histoire de Saint Louis (Paris: Didot, 1874).

Jómsvíkinga SAGA. Olafur Halldórsson, ed., Jómsvíkinga saga [AM 291 $4^{\circ}$ ] (Reykjavík: Jón Helgason, 1969).

N. F. Blake, ed., Jómsvíkinga saga/The Saga of the Jomsvikings [Cod. Holm. $74^{\circ}$ ] (London: Thomas Nelson, 1962).

Jóns SAGa HeLGa. Jón Sigurơsson and Guơbrandur Vigfússon, eds., Biskupa sögur, I (Copenhagen: Møller, 1858).

JORDANES. Theodor Mommsen, ed., Iordanis romana et getica, Monumenta Germaniae historica (Berlin: Weidmann, 1882).

Karlamagnús Saga. C. R. Unger, ed., Karlamagnús saga ok kappa hans (Christiania: H. J. Jensen, 1860).

KJaLNESINGA SAGA. If $F$ XIV (1959).

KöNIG Rother. Theodor Frings and Joachim Kuhnt, eds. König Rother (Halle/Saale: Niemeyer, 1922).

KoRMÁKS SAGA. ÍF VIII (1939).

LANDNÁMABÓK. Í $F$ I (1968).

LAXDELA SAGA: IF V (1934).

Legendary SAGA. Oscar Albert Johnsen, Olafs saga hins helga efter 
pergamenthaandskrift i Uppsala Universitetsbibliotek Delagardieske samling nr. 5 (Oslo: Dybwad, 1922).

Livre D'ArTus. See Vulgate Cycle.

LJósvetNinga SAGa. IF X (1940).

LONGEST SAGA. Olafur Halldórsson, ed., Olás saga Tryggvasonar en mesta, Editiones Arnamagnæanæ, Series A, vols. 1-2 (Copenhagen: Munksgaard, 1958-61).

Malory, Thomas. Eugène Vinaver, ed., The Works of Sir Thomas Malory, 3 vols. (Oxford: Clarendon, 1947).

Marie de France. Alfred Ewert, ed., Lais (Oxford: Blackwell, 1944).

Martianus Capella. De Rhetorica, in Karl Halm, ed., Rhetores latini minores (Leipzig: Teubner, 1863).

MatThew of VendOMe. Ars versificatoria, in Edmond Faral, ed., Les arts poétiques du XII e et du XIII ${ }^{e}$ siècle (Paris: Champion, 1958).

Morkinskinna. Finnur Jónsson, ed., Morkinskinna, Samfund til udgivelse af gammel nordisk litteratur (Copenhagen: Jørgensen, 1932).

Mort Artu. See Vulgate Cycle.

Mǫdruvallabók. Codex Mö̈ruvallensis, Corpus codicum islandicorum medii aevi, 5 [Early Icelandic Manuscripts in Facsimile] (Copenhagen: Levin and Munksgaard, 1933).

Nibelungenlied. Karl Bartsch and Helmut de Boor, eds., Das Nibelungenlied, 20th ed. (Wiesbaden: Brockhaus, 1972).

NJÁLS SAGA. IF XII (1954).

OdDr SNorrason. Finnur Jónsson, ed., Saga Oláfs Tryggvasonar af Oddr Snorrason munk (Copenhagen: Gad, 1932).

Oldest SAGa. Gustav Storm, Otte brudstykker af den ældste saga om Olav den hellige, Norsk historisk kildeskriftfond (Oslo: Grøndahl, 1893).

Orkneyinga SAGa. IF XXXIV (1965).

Parcevals saga. E. Kölbing, Riddarasögur (Strasbourg: Trübner, 1872).

Paul the Deacon. Ludwig C. Bethmann and Georg Waitz, eds., Pauli Historia Langobardorum, Monumenta Germaniae historica, Scriptores rerum langobardicarum et italicarum (Hannover: Hahn, 1878; rpt. 1964).

Prestssaga GuĐmundar góĐa. See Sturlunga saga.

Profectio Danorum. M. Cl. Gertz, ed., De profectione Danorum in Hierosolymam, Scriptores minores historiæ Danicæ medii ævi, 2 vols. (Copenhagen: Gad, 1918-20; rpt. Copenhagen: Munksgaard, 1970), vol. I.

Prophécies de Merlin. Lucy Allen Paton, ed., Les prophécies de Merlin, 2 vols. (New York: D. C. Heath, 1926-27).

Prose Lancelot. See Vulgate Cycle. 
Prose Tristan. Eilert Löseth, Le roman en prose de Tristan, roman de Palamède et la compilation de Rusticien de Pise: Analyse critique d'après les manuscrits de Paris, Bibliothèque de l'Ecole des Hautes Etudes (Paris: Bouillon, 1890).

Queste del saint graal. See Vulgate Cycle.

ReYkdela Saga. IF X (1940).

ROBERT OF BASEvorn. Forma praedicandi, in Th.-M. Charland, ed., Artes praedicandi: Contribution à l'histoire de la rhétorique a u moyen àge (Paris: J. Vrin, 1936).

Leopold Krul O.S.B., tr., "The Form of Preaching," in James J. Murphy, ed., Three Medieval Rhetorical Arts (Berkeley: University of California Press, 1971).

Roman d'Enéas. J.-J. Salverda de Grave, ed., Enéas: Roman $d u X I^{e}$ siècle, 2 vols., Les classiques français du moyen âge, 44 \& 62 (Paris: Champion, 1925-29).

Roman de Troie. Léopold Constans, ed., Le roman de Troie par Benoît de Sainte-Maure, 6 vols., Société des anciens textes français (Paris: Didot, 1904-12).

Saxo Grammaticus. C. Knabe and Paul Herrmann, eds., Saxonis Gesta Danorum, rev. Jørgen Olrik and Hans H. Raeder (Copenhagen: Levin and Munksgaard, 1931).

Scholia VINDOBONENSIA. Joseph Zechmeister, ed., Scholia vindobonensia ad Horatii artem poeticam (Vienna: Apud C. Geroldum Filium Bibliopolam, 1877).

Separate Saga. See Great Saga.

Squire of Low Degree. Walter French and Charles B. Hale, eds., Middle English Metrical Romances (New York: Prentice-Hall, 1930).

Sturlunga saga. Jón Jóhannesson, Magnús Finnbogason, and Kristján Eldjárn, eds. Sturlunga saga, 2 vols. (Reykjavík, 1946).

Sulpitius Victor. Institutiones oratoriae, in Karl Halm, ed., Rhetores latini minores (Leipzig: Teubner, 1863).

SverRIS SAGa. Gustav Ludvig Indreb $\phi$, Sverris saga [AM $3274^{\circ}$ ], Norsk historisk kjeldeskrift-fondet: Skrifter, 46 (Oslo: Dybwad, 1920).

. Finnur Jónsson, ed., Eirspennill [AM 47 fol.], Norsk historisk kildeskriftkommission: Skrifter, 42 (Oslo: Thømte, 1916).

TAsso. Luigi Poma, ed., Torquato Tasso: Discorsi dell' arte poetica e del poema eroico (Bari: Laterza, 1964).

Mariella Cavalchini and Irene Samuel, trs., Discourses on the Heroic Poem (Oxford: Clarendon, 1973).

THEODORICUs. Historia de antiquitate regum Norwagiensium, in Gustav Storm, ed., Monumenta historica Norvegia (Oslo: Brøgger, 1880).

Tномаs. Bartina $\mathrm{H}$. Wind, ed., Les fragments du roman de Tristan, 
poème $d u X^{X I I}{ }^{e}$ siècle, Textes littéraires français, 92 (Geneva: Droz, 1960).

Tristan. See Béroul, Eilhart, and Thomas.

Tristrams saga. Gísli G. Brynjúlfson, ed., Saga af Tristram ok Ísönd, samt Möttuls saga (Copenhagen: Thiele, 1878).

VIE De Saint Alexis. Jean-Marie Meunier, ed., La vie de Saint Alexis, poème français du XI $I^{e}$ siècle (Paris: Droz, 1933).

Viga-Glúms SAGa. IF IX (1956).

Villehardouin. Edmond Faral, ed. and tr., Geoffroi de Villehardouin: La conquête de Constantinople, 2 vols., Les classiques de l'histoire de France au moyen age, 18-19 (Paris: Société d'édition "Les belles lettres," 1938-39).

Vulgate Cycle. Heinrich Oskar Sommer, ed., The Vulgate Version of the Arthurian Romances, 7 vols. + index (Washington: Carnegie Institution, 1908-16).

W. A. Nitze, ed., Le roman de l'estoire dou graal (Paris: Champion, 1927).

Alexandre Micha, ed., Lancelot, roman en prose du XIII ${ }^{e}$ siècle, 3 vols., Textes littéraires français, 247, 249, \& 262 (Paris: Droz, 197879).

Albert Pauphilet, ed., La queste del saint graal: Roman du XIII siècle (Paris: Champion, 1923 and 1949).

. Jean Frappier, ed., La mort le roi Artu: Roman du XIII ${ }^{e}$ siècle, Textes littéraires français (Geneva: Droz, 1964).

P. M. Matarasso, tr., The Quest of the Holy Grail (Harmondsworth, Middlesex: Penguin, 1969).

James Cable, ed., The Death of King Arthur (Harmondsworth, Middlesex: Penguin, 1971).

Voqusunga SAGa. R. G. Finch, ed., Volsunga saga/The Saga of the Volsungs (London: Thomas Nelson, 1965).

Wolfram von Eschenbach. Albert Leitzmann, Wolfram von Eschenbach: Parzival, Altdeutsche Textbibliothek, 12-14 (Halle: Niemeyer, 1902-3).

Helen M. Mustard and Charles E. Passage, trs., Wolfram von Eschenbach: Parzival (New York: Vintage, 1961).

PóRdar saga kakala. See Sturlunga saga.

Porsteins SAGa hVITA. IF XI (1950).

Porsteins pátTr stangarhogGs. Í $F$ XI (1950). 
